$28 \mathrm{MeV}$ and $32 \mathrm{MeV}$-energies close to and slightly above the Coulomb barrier for the interaction of these oxygen nuclei. Gelbke and his colleagues found that at these energies the angular distribution of the scattered particles has two distinct minima in intensity between $90^{\circ}$ and $150^{\circ}$; these occurred at approximately the same angle regardless of the energy of the incident particle. This observation immediately rules out explanations of the anomalous behaviour based on the absorption of the incident particle by the nucleus to different extents depending on its angular momentum relative to the scattering nucleus. Thus the anomalous behaviour cannot be explained by an optical model that contains a term which explicitly depends on the angular momentum of the particles, although observations at one energy can be adequately described by such a model.

Gelbke et al. think that the incident ${ }^{16} \mathrm{O}$ nucleus picks up the two least bound neutrons in an ${ }^{18} \mathrm{O}$ nucleus and imparts sufficient momentum to the ${ }^{16} \mathrm{O}$ nucleus thus formed for it to emerge from the target. This ${ }^{16} \mathrm{O}$ is, of course, indistinguishable from a normally scattered ${ }^{16} \mathrm{O}$ nucleus.

The experimental angular distributions are fitted by a model which includes contributions from such an exchange mechanism and, as well as reproducing each individual measured distribution at the three energies, the variation with energy is also satisfactorily reproduced. Minor differences in the theoretical fits to the experimental points at the three energies are attributed to the fact that the probability of the incident ${ }^{16} \mathrm{O}$ nucleus picking up the two neutrons from ${ }^{18} \mathrm{O}$ varies with energy.

\section{RADIATION DAMAGE}

\section{Precipitate Instability}

from our Materials Science Correspondent IT is not at all surprising that an unprecedented research effort is being devoted to a form of radiation damage that is specific to fast reactors for, even though nuclear power, under pressure from environmentalists, is unfashionable, those persons responsible for national energy policy know perfectly well that in the long run most power must come from nuclear reactors, and the fast reactor in particular.

Radiation damage in fast reactors is a consequence of the fast neutrons and the high fuel temperatures: fuel swelling is caused by the formation of voids, which are themselves formed by the selective agglomeration of excess lattice vacancies resulting from neutron damage. When a neutron starts a collision sequence which causes a number of atoms to be knocked out of the crystal lattice, equal numbers of vacancies and interstitials result. If, as is all too apt to happen, the interstitial atoms migrate faster than the vacancies, then the former quickly disappear at "sinks" and the vacancies are left high and dry; they are then likely to combine to form microscopic cavities or voids, and the fuel or the material of the fuel envelope swells dangerously. This has been a serious problem, but because of extensive research since 1967 it is now well on the way to solution.

One of the principal remedies is to use alloys containing a fine dispersion of precipitates; migrating interstitials are in some way arrested when they impinge on a precipitate particle, and are held captive there until they are neutralized by a wandering vacancy. So, in the end, almost all the interstitials recombine with vacancies and few vacancies are left to form voids. Thus, some nickel-base alloys of the Nimonic type have been found particularly suitable for the manufacture of fuel cans, for the fine precipitates of $\gamma^{\prime}$ (ordered $\mathrm{Ni}_{3} \mathrm{Al}$ ) impede swelling in the manner described. For this purpose, it is necessary for the precipitates to be particularly finely distributed (to increase the probability of interstitial capture), and to stay that way in service. It is this last requirement which is difficult, because all precipitate populations are subject to the law of Ostwald ripening -that is, operation of the Matthew Principle, or the growth of the large at the expense of the small.

A group of investigators at Harwell have now systematically examined, both theoretically and experimentally, the behaviour of precipitate populations in a radiation field. The Matthew Principle is counteracted by the steady destruction of precipitates by neutron damage, and the diffusion of solute atoms into the matrix at a rate which is itself accelerated by the presence of excess vacancies. From their theoretical calculations, Nelson, Hudson and Mazey (J. Nucl. Mater., 44, 318 ; 1972) find that bigger particles redissolve faster than small ones, and that this is true whichever of two alternative mechanisms of resolution operates.

The final conclusion is that in the presence of steady neutron irradiation, large particles dissolve preferentially but small particles on balance still grow, so that a dynamic equilibrium is established and most particles finish up close to a preferred size, which is quite small. Irradiation thus effectively neutralizes Ostwald ripening. This conclusion is confirmed by experimental observations on a Nimonic-type alloy containing coherent $\gamma^{\prime}$ particles, but not by observations on another kind of nickel alloy containing incoherent precipitates. The last of these are subject to normal coarsening, because (it seems) one of the mechanisms for irradiation induced resolution of precipitates, which requires mass transport across the particle/matrix interface, does not work satisfactorily across incoherent (high energy) interfaces.

\section{BL LAC OBJECTS}

\section{Link with Galaxies?}

by our Cosmology Correspondent

BL LACERTAE is the archetype of a class of interesting extragalactic objects. These BL Lac objects seem to be exceedingly compact, and are in many ways reminiscent of QSOs, with the important difference that they have no spectral lines and thus no measured redshifts. Although only half a dozen of the objects are known, and perhaps no more than three can be confidently said to be of the same family as BL Lac itself, they have recently been the subject of intensive study because they may be related to QSOs.

One interesting speculation-it is no more at present-is that BL Lac objects may be blueshifted QSOs; their flat,

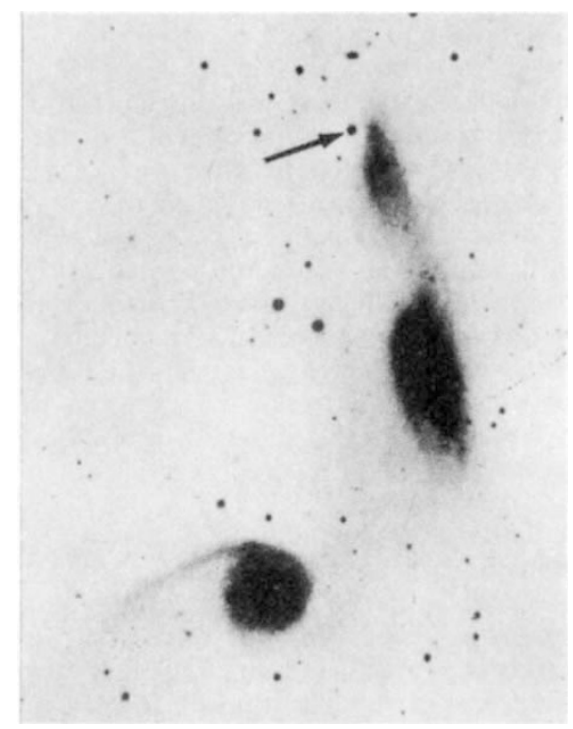

NGC 2993 (bottom), NGC 2992, and Weedman 2 (arrow). (Plate obtained by H. C. Arp.)

line-free spectra could well represent a region "normally" seen in the infrared. This idea is important, even though the sample of BL Lac objects is so small, because the definite detection of even one blueshifted QSO would have a significant bearing on the "redshift/distance relation" of QSOs. There is mounting evidence that the redshift of QSOs cannot be explained simply as a Doppler effect of motion in an expanding universe, and that part of the redshift, in some QSOs at least, must arise in some other way.

Paramount among the reasons underlying this view is the discovery of some 